\title{
THE TURBULENT FLOW ALONG A SEMI-INFINITE PLATE*
}

\author{
BY \\ P. Y. CHOU \\ National Tsing Hua University, Peiping, China
}

1. Introduction. The investigation of the turbulent flow along a semi-infinite plate is of both theoretical and technical interest, as it leads to the magnitude of the skin-friction acting upon the plate at high Reynolds numbers. Interpretations of the velocity distribution within the tubulent boundary layer have been given by Howarth ${ }^{1}$ according to the transport theories. But the formula that fits experiment best is von Kármán's logarithmic law of velocity distribution which is a consequence of his similarity theory of turbulence.

The purpose of the present paper is to show that von Kármán's velocity distribution law is consistent with the program of treating turbulent flows developed by the author. ${ }^{2}$ For the solution of the present problem we shall adopt the following three conditions and simplifications.

(1) We shall use the law of dynamical similarity down-stream along the flow. which has been verified by Dryden's experiments. The mean velocity along the direction of the undisturbed flow $U$ which is parallel to the plane of the plate when expressed in units of the friction velocity $U_{\tau}$ has been found by Dryden to be a function of the dimensionless variable $\eta=y U_{\tau} / \nu$ in which $y$ is the coordinate perpendicular to the plate and $U_{\tau}$ is a function of $x$, the distance along the plate from its leading edge. We now generalize this result by assuming that the mean pressure $\bar{p}$ and all the other velocity correlations when expressed in units of proper powers of $U_{\tau}$ are dimensionless functions of $\eta$ only.

(2) Reynolds' equation for mean motion and the equations of double and triple correlations will be used. In the equations of triple correlation we shall disregard the quadruple correlations. The range of validity of this approximation and the possibility of its experimental verification will be discussed in Sec. 5 .

(3) We shall assume the constancy of the micro-scale of turbulence $\lambda$ and of the squares of components of velocity fluctuation across the boundary layer. This condition is used for mathematical simplicity. It is, however, consistent with the result to be shown below that the shearing stress is constant across the boundary layer and can therefore be regarded as a condition of similarity. On the other hand, as will be seen presently, the variation of the mean squares of velocity fluctuation can be accounted for, though their solution will be rather complicated. This part of calculation is omitted due to lack of measurements.

The logarithmic law of temperature distribution in the boundary layer of a heated plate can also be established by the present method of approach to the turbulence problem and its solution will be indicated at the end of the paper.

2. Reynold's equations of mean motion. Let the positive half of the $x z$-plane represent the given semi-infinite plane and the leading edge of the plane coincide with the $z$-axis, the $y$-axis being perpendicular to the plane. Using $U$ and $V$ to denote the

* Received Oct. 21, 1946.

${ }^{1}$ S. Goldstein, Modern developments in fluid dynamics (1938) pp. 361-371.

2 P. Y. Chou, Quart. Appl. Math. 3, 38-54 (1945). 
$x$ - and $y$-components of the mean velocity, we find the Reynolds' equations of mean motion to be

$$
\begin{aligned}
& U \frac{\partial U}{\partial x}+V \frac{\partial U}{\partial y}=\frac{1}{\rho} \frac{\partial \tau_{x y}}{\partial y} \\
& U \frac{\partial V}{\partial x}+V \frac{\partial V}{\partial y}=-\frac{1}{\rho} \frac{\partial \bar{p}}{\partial y}+\frac{1}{\rho} \frac{\partial \tau_{y y}}{\partial y}
\end{aligned}
$$

in which both the viscous stress and the terms in components of turbulent stress involving partial differentiations with respect to $x$ on the right hand sides of Eqs. (2.1) and (2.2) are neglected. This is permissible on account of the thinness of the turbulent boundary layer.

To satisfy the equation of continuity, we introduce a stream function $\Psi$ as usual and write

$$
U=\frac{\partial \Psi}{\partial y}, \quad V=-\frac{\partial \Psi}{\partial x} .
$$

It is well-known that by integrating Eq. (2.1) across the boundary layer, we find the turbulent shearing stress on the plate. Thus, ${ }^{3}$

$$
\tau_{0}=\left.\tau_{x y}\right|_{y=0}+\rho U_{\tau}^{2}=\frac{\partial}{\partial x} \int_{0}^{\delta} \rho\left(U_{1}-U\right) U d y,
$$

where $U_{\tau}$ is the friction velocity, $\delta$ the thickness of the layer and $U_{1}$ the velocity in the free stream outside the layer. Hence if the mean velocity $U$, as a function of $y$, were known, Eq. (2.4) would define the friction velocity $U_{\tau}$ as a function of $x$.

Here we introduce Dryden's experimental result that the ratio of the mean velocity $U$ and $U_{\tau}$ is a function of the dimensionless variable $\eta$, where $\eta$ is defined by

$$
\eta=y U_{\tau} / \nu .
$$

In other words, the stream function $\Psi$ is given by

$$
\Psi=\nu F(\eta)
$$

so that according to equation (2.3)

$$
U=U_{\tau} F^{\prime}(\eta)
$$

This relation can be construed as the condition of dynamical similarity in the different cross-sections of the boundary layer. From Eqs. (2.3) and (2.6), we obtain

$$
V=-y F^{\prime} \frac{\partial U_{\tau}}{\partial x}
$$

Let us assume that dynamical similarity also holds true for the shearing stress, i.e. that

$$
-\frac{1}{\rho} \tau_{x y}=U_{\tau}^{2} f_{4}(\eta)
$$

${ }^{8}$ S. Goldstein, loc. cit., p. 132. 
Substituting from Eqs. (2.7), (2.8) and (2.9) into the equation of mean motion (2.1), we obtain

$$
F^{\prime 2} \frac{\partial U_{\tau}}{\partial x}=-\frac{U_{\tau}^{2}}{\nu} f_{4}^{\prime} .
$$

We may estimate the order of magnitude of $\partial U_{\tau} / \partial x$ by means of von Kármán's coefficient of local skin friction in the following way: ${ }^{1}$

$$
\frac{d z}{d x}=\frac{1}{x} \frac{1}{1+2 / z}
$$

where $z=K U_{1} / U_{\tau}, K=0.4$.

The derivative $d z / d x$ thus tends to $1 / x$ for large values of $z$, Hence equation (2.10) becomes

$$
f_{4}^{\prime}=\frac{F^{\prime 2}}{K} \frac{\nu}{U_{1} x}=F^{\prime 2} / K R_{x} .
$$

The Reynolds number $R_{x}$ in Dryden's measurements ${ }^{4}$ is of the order of $10^{6}$ and $F^{\prime}$ is around 20. This shows that $f_{4}^{\prime}$ is of the order $10^{-3}$ and can be neglected. In short, $f_{4}$ or the shearing stress is essentially constant across the turbulent boundary layer.

3. Equations of double and triple correlations, the mean velocity distribution. We suppose that the law of dynamical similarity not only holds true for the mean velocity distribution, but also for the double and triple velocity correlations as well. Hence we write

$$
\begin{aligned}
& -\frac{1}{\rho} \bar{p}=U_{\tau}^{2} f_{0}(\eta) \\
& -\frac{1}{\rho} \tau_{x x}=U_{\tau}^{2} f_{1}(\eta), \quad-\frac{1}{\rho} \tau_{y y}=U_{\tau}^{2} f_{2}(\eta), \quad-\frac{1}{\rho} \tau_{z z}=U_{\tau}^{2} f_{3}(\eta), \\
& -\frac{1}{\rho} \tau_{x y}=U_{\tau}^{2} f_{4}(\eta) \\
& \left.\begin{array}{rlrl}
\overline{W_{x}^{3}} & =U_{\tau}^{3} h_{0}(\eta), & \overline{W_{x}^{2} W_{y}} & =U_{\tau}^{3} h_{1}(\eta), \\
\overline{W_{x} W_{y}^{3}} & =U_{\tau}^{3} h_{2}(\eta), & \overline{W_{y}^{2}} & =U_{\tau}^{3} h_{3}(\eta), \\
\overline{W_{x} W_{z}^{3}} & =U_{\tau}^{3} h_{4}(\eta), & \overline{W_{y} W_{z}^{2}} & =U_{\tau}^{3} h_{5}(\eta) .
\end{array}\right\}
\end{aligned}
$$

Since our interest at present lies mainly in the mean velocity distribution, we shall only put down the equations which are pertinent for its determination. When written in full the component equation $(i=1, k=2)$ for the double correlation ${ }^{2}$ is

$$
\begin{aligned}
-\frac{1}{\rho}\left[\tau_{x x} \frac{\partial V}{\partial x}+\tau_{y y} \frac{\partial U}{\partial y}\right] & -\frac{1}{\rho} U \frac{\partial \tau_{x y}}{\partial x}-\frac{1}{\rho} V \frac{\partial \tau_{x y}}{\partial y} \\
+ & \frac{\partial}{\partial x} \overline{w_{x}^{2} w_{y}}+\frac{\partial}{\partial y} \overline{w_{x} w_{y}^{2}}=-a_{2112} \frac{\partial U}{\partial y}-\frac{2 \nu k_{\overline{\lambda^{2}}}}{w_{x} w_{y}} .
\end{aligned}
$$

${ }^{4}$ H. L. Dryden, N. A. C. A. Report No. 562 (1936). 
In the above equation we have only retained the largest term $a_{2112} \partial U / \partial y$ among the sum $a_{m t k}^{n} U^{m}, n$.

Next, introduce the substitutions (2.7), (2.8), (3.1) and (3.2) into Eq. (3.3). Obviously, the terms involving $\partial V / \partial x$ and $\overline{w_{x}^{2} w_{y}}$ in (3.3) are small. With the use of the relation (2.11), we find that

$$
-\frac{1}{\rho} U \frac{\partial \tau_{x y}}{\partial x}-\frac{1}{\rho} V \frac{\partial \tau_{x y}}{\partial y}=-\frac{2 U_{\tau}^{4}}{K x U_{1}} F^{\prime} f_{4} .
$$

The appearance of $x U_{1}$ in the denominator means that this term contains the reciprocal of the Reynolds number $R_{x}$ as a factor when compared with the remaining terms, says, $-\tau_{y y} \partial U / \rho \partial y$, in the equation. Hence as a good approximation of (3.3) we have

$$
-a F^{\prime \prime}+h_{2}^{\prime}=-l_{0} f_{4}
$$

where $l_{0}$ and $a$ are defined by

$$
l_{0}=2 k \nu^{2} / \lambda^{2} U_{\tau}^{2}, \quad a=-\left(f_{2}+a_{2112} / U_{\tau}^{2}\right) .
$$

The fact that only the terms in $\partial U / \partial y$ and $\partial \overline{w_{x} w_{y}^{2}} / \partial y$ on the left-hand side of equation (3.3) remain can be interpreted that $V$ is negligible and all the functions $U, \tau_{i j}$ and $\overline{w_{i} w_{j} w_{k}}$ depend upon $\eta$ alone and are very approximately independent of $x$, the coordinate from the leading edge of the plate. An equivalent statement is that the turblent boundary layer is very thin. This simplifies the problem; its mathematics becomes analogous to that for the problem of mean velocity distribution in a channel. ${ }^{5}$

The component equation $(i=1, k=1,1=2)$ of the equations of triple correlation which is needed for the determination of the mean velocity distribution can be obtained by the method used in deriving equation (3.5) as:

$$
2 \frac{\partial U}{\partial y} \overline{w_{x} w_{y}^{2}}+\frac{\partial}{\partial y} \overline{w_{x}^{2} w_{y}^{2}}=-b_{21112} \frac{\partial U}{\partial y}-c_{112}+\frac{1}{\rho^{2}}\left(2 \tau_{x y} \frac{\partial \tau_{x y}}{\partial y}+\tau_{x x} \frac{\partial \tau_{y y}}{\partial y}\right) .
$$

It is obvious that the quadruple correlation term $\partial \overline{w_{x} w_{y}^{2}} / \partial y$ must be of the same order of magnitude as $\tau_{x x} \partial \tau_{y y} / \rho^{2} \partial y$ and we shall now neglect these quantities against the term $\partial U / \partial y\left(2 \overline{w_{x} w_{y}^{2}}+b_{2112}\right)$. This is equivalent to the statement that we limit our solution to the region where the following inequality holds true

$$
\left|\frac{\partial U}{\partial y}\left(2 \overline{w_{x} w_{y}^{2}}+b_{21122}\right)\right|>\left|\overline{w_{x}^{2}} \frac{\overline{\partial w_{y}^{2}}}{\partial y}-\frac{\partial}{\partial y} \overline{w_{x}^{2} w_{y}^{2}}\right| .
$$

Apparently this condition can be satisfied in the middle of the layer where $\partial U / \partial y$ is large, while on its outer edge it may break down as we shall see in Sec. 5. The quantity $c_{112}$ in (3.7) will be considered as independent of $\eta$ because it varies but slowly with the coordinates, as has been proved previously. ${ }^{2}$ The derivative $\partial \tau_{x y} / \partial y$ is small according to the relation (2.12) and can therefore be neglected. Introducing expressions (2.7) and (3.2) into Eq. (3.7) and writing

$$
c_{112}=2 c U_{\tau}^{5} / \nu
$$

5 P. Y. Chou, Quart. of Appl. Math., 3, 198-209 (1945). 
we find, after using the inequality (3.8),

$$
h_{2}=-c / F^{\prime \prime}-b_{21112} / 2 U_{\tau}^{3}
$$

Like $c_{112}$ we take $b_{2112} / U_{\tau}^{3}$ to be also a constant.

Elimnation of the variable $h_{2}$ between equations (3.5) and (3.9) yields the final equation for the determination of means velocity distribution,

$$
a F^{\prime \prime}+c \frac{d}{d \eta} \frac{1}{F^{\prime \prime}}=l_{0} f_{4}
$$

Since the variation of $f_{4}$ with $\eta$ across the boundary layer is small, we assume that $a$ which depends upon $\frac{w_{y}^{2}}{2}$ defined by equation (3.6) is also a constant. There is no $a$ priori experimental justification for the assumption, but it simplifies the calculation a great deal. As a matter of fact the determination of the behaviour of $\overline{w_{x}^{2}}, \overline{w_{y}^{2}}$ and $\overline{w_{z}^{2}}$ within the layer is not wholly impossible by means of the rest of the equations of double and triple correlations which have not been written out explicitly,* though the work involved will be rather complicated. For the same reason of simplifying calculation we take $l_{0}$ to be constant, namely, $\lambda$ to be independent of $\eta$ and leave out the equation of vorticity decay. ${ }^{2}$

Integrating the equation (3.10) twice, we find

$$
\eta=\frac{a}{l_{0} f_{4}} F^{\prime}+A\left(e^{l_{0} f_{4} F^{\prime} / c}-1\right)
$$

where $A$ is a constant of integration. The other arbitrary constant has been determined by the condition on the surface of the plate: $\eta=0, F^{\prime}=0$. Another constant of the remaining three in equation (3.11) can be disposed of, as a consequence of the linear dependence of the mean velocity $U$ upon $\eta$ near the plate, by requiring that it become $\eta=F^{\prime}$, when $\eta$ is small. On the other hand for large values of $\eta$ the mean velocity $U$ varies with $\eta$ logarithmically, confirming von Kármán's result. For this latter reason we take the factor of $F^{\prime}$ in the exponential function to be 0.4 , when we compare formula (3.11) with Dryden's measurements. ${ }^{4}$ The last constant $A$ is determined by passing the theoretical curve through the mean experimental value of $F^{\prime}=18.2$ at $\log _{10} \eta=2.2$. The result can be summarized in the equation

$$
\eta=0.959 \frac{U}{U_{\tau}}+0.1024\left(e^{0.4 U / U_{\tau}}-1\right) .
$$

The velocity distribution given above resembles that within the channel ${ }^{5}$ in its functional dependence upon $\eta$, though the values of the corresponding constants appearing in the two cases are different as is to be expected. Comparison between theory and experiment is given in Fig. 1. Their agreement is well-known to be satisfactory.

* They will be the same as Eqs. (2.3), (2.5), (2.6), (3.1), (3.3) and (3.4) in the paper quoted in Footnote 5 . 


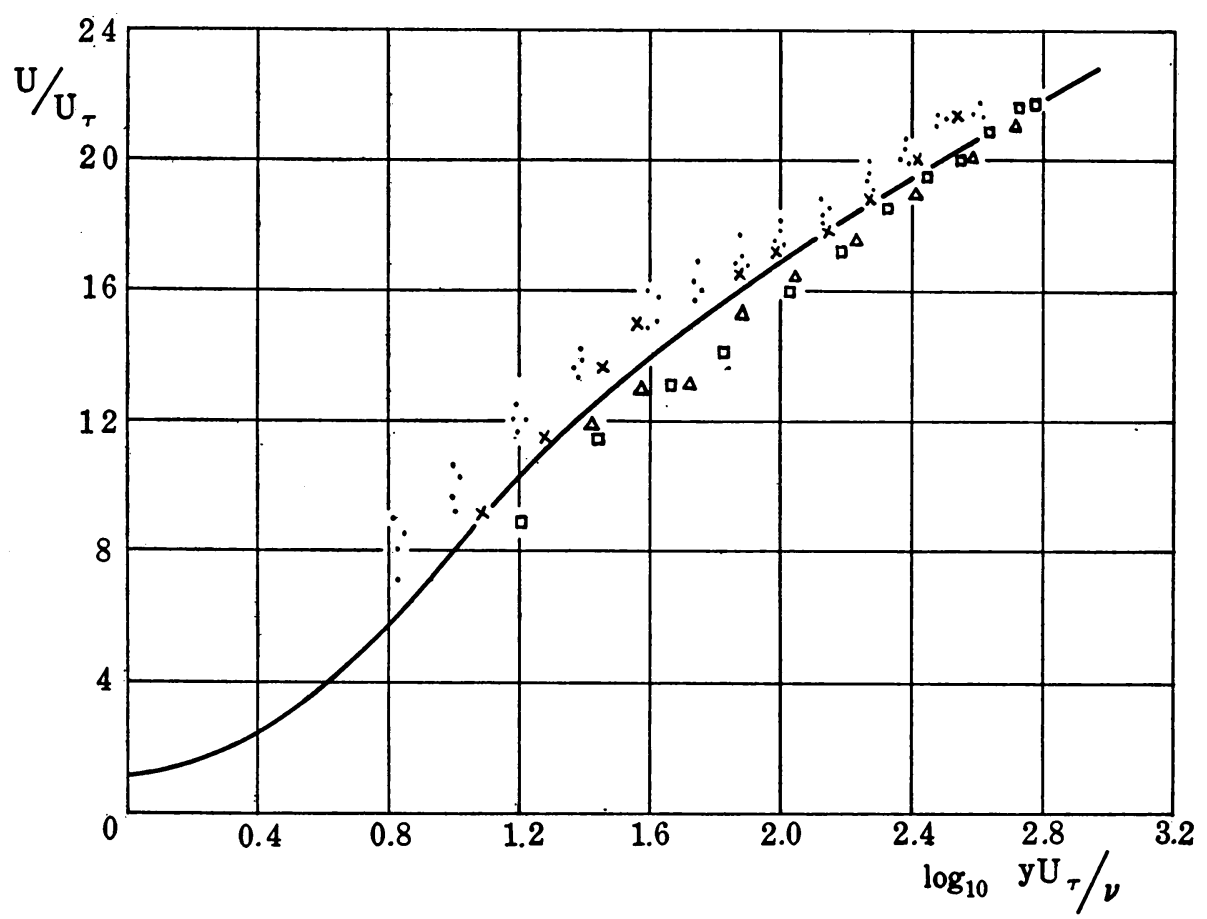

FIG. 1

Velocity distribution in a turbulent boundary layer.

Theory:

Dryden's experimental results:

$$
\begin{aligned}
& \cdot \quad R_{x}=335,000, \square R_{x}=507,000, \\
& \times R_{x}=788,000, \triangle R=983,000, \\
& x \text { being measured from the transition point. }
\end{aligned}
$$

4. The coefficient of local skin-friction. The explicit relation between the coefficient of local skin-friction $c_{f}$, which is defined by

$$
c_{f}=\tau_{0} / \frac{1}{2} \rho U_{1}^{2}=U_{\tau}^{2} / \frac{1}{2} U_{1}^{2},
$$

and the Reynolds number $R_{x}=U_{1} x / \nu$ can now be determined by substituting the mean velocity distribution (3.12) into Eq. (2.4) and integrating. The thickness of the boundary layer $y=\delta$ is found by setting $U=U_{1}$ in Eq. (3.12). Retaining the large quantities in the final result after integration, we find

$$
\frac{d R_{x}}{d z}=1.6 e^{z}\left(z^{2}-2 z+2\right)
$$

where $z$ has been defined in Eq. (2.11) as $0.4 U_{1} / U_{\tau}$. Integrating (4.2) again gives

$$
R_{x}=1.6 e^{z}\left(z^{2}-4 z+6\right)-6 .
$$

The constant of integration is determined by $x=0$, when $z=0$, under the assumption that the flow is turbulent from the leading edge. 
For large $z$ we keep only the $z^{2}$ term on the right member of equation (4.3) and the result can be put in the form

$$
c_{f}^{-1 / 2}=1.19+4.08 \log _{10}\left(R_{x} c_{f}\right) .
$$

The two constants appearing above according to Kempf's experiments are 1.7 and 4.15 respectively. ${ }^{1}$ The form of $R_{x}$ given in (4.3) also justifies our use of the relation (2.11) which leads to the very approximate constancy of the shearing stress across the boundary layer as has been demonstrated by relation (2.12).

5. Some further experimental verifications and validity of the solution. The present analysis shows that, according to (2.12), the shearing stress is constant across the turbulent boundary layer, if the Reynolds number of the flow is sufficiently high. This result can be subject to direct experimental proof, since $\tau_{x y}$ at different points of the fluid is now measurable by the hot-wire technique. On the other hand to establish the validity of the inequality (3.8) experimentally is probably not simple. There is a greater chance of its fulfillment, if $\overline{w_{x}^{2}}$ and $\overline{w_{y}^{2}}$ are also constant across the layer. This constancy of $\overline{w_{x}^{2}}$ and $\overline{w_{y}^{2}}$ can be interpreted as the consequence of the condition of "similarity" mentioned before. On the other hand, even if this requirement of similarity were satisfied, it has its limitations and we can determine the validity of the present solution by considering the values of the constants appearing in equations (3.11) and (3.12).

Since $f_{4}$ is independent of $\eta$, according to its definition (2.9) and relation (2.4) it must be -1 . From Eqs. (3.11) and (3.12) we find

$$
a / l_{0}=-0.959, \quad l_{0} / c=-0.4 \text {. }
$$

The quantity $l_{0}$ is determinable by using Eq. (3.6) with the micro-scale of turbulence $\lambda$, which comes from correlation measurements and $k$, a constant, which is present in all the three equations for $\overline{w_{x}^{2}}, \overline{w_{y}^{2}}$, and $\overline{w_{z}^{2}}$ and is also observable. Thus both $a$ and $c$ are known after $l_{0}$ is fixed.

The factor $1 / F^{\prime \prime}$ in equation (3.9) for $h_{2}$ varies according to (3.12) between unity, when $U / U_{\tau}=F^{\prime}=0$ on the plate and about 120 when $F^{\prime}=20$. Hence the part of $h_{2}$ variable with $\eta$ can take the extreme values of $-c$ and $-120 c$. According to its definition in (3.2), the value of $h_{2}$ can not be very large. This means that there must be an upper limit of $F^{\prime}$, namely, the outer limit of the boundary layer within which the relation (3.9) is val:d. Again if triple velocity correlations were measurable by the hot wire technique, to test this relation directly within its region of validity should be an interesting problem.

The foregoing analysis shows the important rôle played by the triple velocity correlation within the turbulent boundary layer. This can be interpreted as indicating that turbulence is generated in this layer and transported away from it. The triple velocity correlation is a measure of this transport; hence the set of equations governing its behaviour is needed for the interpretation of the mean velocity distribution. Indeed, if this interpretation were correct, it is easy to understand that only the equations of mean motion and of double correlation with the omission of the triple correlation terms in the latter are insufficient to explain the velocity distribution within the boundary layer, for this simplified set of the equations of double correlation only tells us the balance between the production of turbulent energy by deformation of the mean flow and the decay of turbulence. Although this picture 
seems to give a good account of the velocity distributions in the center of the channel and in free turbulence like jets and wakes, it is definitely insufficient for the phenomena dealt with in the present paper.

The present paper is obviously incomplete in the sense that theoretical computations of the mean squares of turbulent fluctuations are not given. It does show, however, that a certain type of function for the mean velocity distribution is necessary. Though refinements of calculation and measurements may change this result to some extent, the main feature of the function will probably remain the same. This point has been borne out by the corresponding calculation of the velocity distribution in the channel under the assumption of different turbulent levels. ${ }^{5}$

The temperature distribution within the boundary layer of a heated plate can be calculated by means of the equations of mean temperature and of the double and triple velocity and temperature correlations. We obtain an equation for the mean temperature distribution analogous to (3.11), and it agrees with the measurements of Éliás. ${ }^{6}$ Obviously a limitation on the validity of the solution similar to (3.8) is also present.

${ }^{6}$ F. Eliás, Zeits. angew. Math. Mech., 10, 1-14 (1930). 